\title{
Quantum ring models and action-angle variables
}

\author{
Stefano Bellucci ${ }^{a}$, Armen Nersessian $^{b}$, Armen Saghatelian $^{b}$ and Vahagn Yeghikyan ${ }^{b}$ \\ ${ }^{a}$ INFN-Laboratori Nazionali di Frascati, Via E. Fermi 40, 00044 Frascati, Italy \\ ${ }^{b}$ Yerevan State University, 1 Alex Manoogian St., Yerevan, 0025, Armenia
}

\begin{abstract}
We suggest to use the action-angle variables for the study of properties of (quasi)particles in quantum rings. For this purpose we present the action-angle variables for three two-dimensional singular oscillator systems. The first one is the usual (Euclidean) singular oscillator, which plays the role of the confinement potential for the quantum ring. We also propose two singular spherical oscillator models for the role of the confinement system for the spherical ring. The first one is based on the standard Higgs oscillator potential. We show that, in spite of the presence of a hidden symmetry, it is not convenient for the study of the system's behaviour in a magnetic field. The second model is based on the so-called $C P^{1}$ oscillator potential and respects the inclusion of a constant magnetic field.
\end{abstract}

\section{Introduction}

It is well-known that, for the systems with finite motion, one can introduce the distinguished set of phase space variables (the "action-angle" variables), such that the "angle" variables parameterize the torus, while their conjugated "action" variables are the functions of constants of motions only [1. As a consequence, the Hamiltonian depends only on action variables. The formulation of the integrable system in these variables gives us a comprehensive geometric description of its dynamics. Such a formulation defines a useful tool for the developing of perturbation theory, since the "action" variables define adiabatic invariants of the system. The action-angle formulation is important from the quantum-mechanical point of view as well, since in actionangle variables the Bohr-Sommerfeld quantization is equivalent to the canonical quantization, with trivial expressions for the wavefunctions. Hence, evaluation of quantum-mechanical aspects of such system becomes quite simple in this approach.

So, the action-angle variables form a useful tool for the study of systems with finite motion. But just such systems presently attract much attention because of progress in mesoscopic physics, where we usually deal with a motion of (quasi)particles localized in quantum dots, quantum layers etc. On the other hand, due to the recent progress in nanotechnology, now the fabrication of various low-dimensional systems of complicated geometric form (nanotubes, nanofibers, spherical and cylindrical layers) become possible [3]. In these context the methods of quantum mechanics on curved space should be relevant for the description of the physics of nanostructures. Say, the common method for the localizing of the particle in the disc or in the cylinder is that of the two-dimensional oscillator for the role of the confinement potential. Similarly, for the localization of the particle quantum lens (e.g. GaAs/In $n_{1-x} G a_{x} A s$, see [4) one can use the Higgs model of the spherical oscillator defined by the potential $V_{\text {Higgs }}=\frac{1}{2} \omega^{2} r_{0}^{2} \tan ^{2} \theta$ [5]. Another confinement potential which could be used for the localization of the (quasi)particles in quantum lens, is the potential of the so-called $C P^{1}$ oscillator $V_{\text {Higgs }}=2 \omega^{2} r_{0}^{2} \tan ^{2} \theta / 2[6$. The advantage of the latter potential is with respect to the magnetic field, which has a constant magnitude on the surface of the sphere. Such a magnetic field is precisely the magnetic field of a Dirac monopole located at the center of sphere. So, formally this field is an unphysical one. However, due to the restriction of the electron in the segment/ring of the spherical layer, it could be viewed as a physical field generated e.g. by the pole of a magnetic dipole. The fabrication of semiconductor ring-shaped systems [7, presently referred to as quantum rings (e.g. In $(G a) A s$ - two-dimensional quantum rings), led to the use of the singular oscillator potential with the role of the confinement one. A pioneering work on the theoretical study of the impact of the magnetic field on the electron properties in a quantum ring was written by Chakraborty and Pietelainen [8]. There, for the role of the confinement potential restricting the motion of electrons in the quantum ring the shifted oscillator potential $V_{\mathrm{ChP}}=\beta\left(r-r_{0}\right)^{2}$ was choosen. The results obtained within this approximation are in a good correspondence with experimental data. The quantum ring model of Chakraborty and Pietelainen is not exactly solvable in the general case, and it assumes 
the use of numerical simulations. The quantum ring model based on the singular oscillator system 9 has been suggested as an analytically solvable alternative to the Chakraborty-Pietelainen model. Although calculations performed within the Chakraborty-Pietelainen model are in better correspondence with experimental data than those within the singular oscillator potential [10, the latter has its own place in the study of quantum rings (see,e.g. 11]).

In analogy with the above models, one can suppose, that singular versions of two-dimensional Higgs and $C P^{1}$ oscillators may be appropriate candidates for the confinement potential localizing the motion of the electron in the ring of a spherical quantum layer.

By the above listed reasons we present, in this paper, the action-angle formulation of the two-dimensional singular oscillator and of its spherical generalizations based on Higgs and $C P^{1}$ spherical oscillator models.

We shall start from the simple model of a two-dimensional singular oscillator given by the Hamiltonian

$$
H=\frac{\mathbf{p}^{2}}{2}+\frac{\alpha^{2}}{2 \mathbf{r}^{2}}+\frac{\omega^{2} \mathbf{r}^{2}}{2} .
$$

Then we shall consider a two-dimensional singular spherical oscillator defined by the following Hamiltonian:

$$
H_{\mathrm{Higgs}}=\frac{p_{\theta}^{2}}{2 r_{0}^{2}}+\frac{p_{\varphi}^{2}}{2 r_{0}^{2} \sin ^{2} \theta}+\frac{\alpha^{2}}{2 r_{0}^{2}} \cot ^{2} \theta+\frac{\omega^{2} r_{0}^{2}}{2} \tan ^{2} \theta,
$$

where $r_{0}$ is the radius of the sphere.

This system generalizes the well-known Higgs model of the spherical oscillator [5, whose uniqueness is in the closeness of all trajectories, which reflects the existence of a number of hidden symmetries equal to the those of the Euclidean oscillator. By this reason, the Higgs oscillator is a convenient background for the developing of perturbation theory. Particularly, it admits the anisotropic modification preserving the integrability of the system [12. Hence, such a model of the spherical ring should be convenient for the study of electrons behavior in external potential fields, e.g., in the electric one. However, it is easy to observe that the (singular) Higgs oscillator does not preserve its exact solvability in the presence of a constant magnetic field, in contrast with the Euclidean one. While the study of quantum dot systems in a magnetic field is of a special physical importance. By this reason we consider the alternative model of the singular spherical oscillator, given by the Hamiltonian 13

$$
H_{C P^{1}}=\frac{p_{\theta}^{2}}{2 r_{0}^{2}}+\frac{p_{\varphi}^{2}}{2 r_{0}^{2} \sin ^{2} \theta}+\frac{\alpha^{2}}{8 r_{0}^{2}} \cot ^{2} \frac{\theta}{2}+2 \omega^{2} r_{0}^{2} \tan ^{2} \frac{\theta}{2} .
$$

It is based on the model of the oscillator on complex projective spaces [6] and, in contrast with the (singular) Higgs oscillator, it respects the inclusion of a constant magnetic field (of the Dirac monopole). Let us notice that a similar model on the four-dimensional sphere and hyperboloid respects the inclusion of the BPST instanton field [14. Quantum mechanical solutions of (1.3) are not constructed yet. But they could be found by a proper modification of the solutions of the corresponding non-singular system (third reference in [6]). Because of the absence of hidden symmetries, this model is not convenient for the study of the system in external potential (e.g. electric ) fields. But it convenient for the study of the interaction with the external magnetic field.

\section{Action-angle variables}

The well-known Liouville theorem gives the exact criterium of integrability of the $N$-dimensional mechanical system: that is the existence of $N$ mutually commuting constants of motion $F_{1}=H, \ldots, F_{n}:\left\{F_{i}, F_{j}\right\}=$ $0, i, j=1, \ldots N$. The theorem also states that if the level surface $M_{f}=\left(\left(p_{i}, q_{i}\right): F_{i}=\right.$ const $)$ is a compact and connective manifold, then it is diffeomorphic to the $N$-dimensional torus $T^{N}$. The natural angular coordinates $\boldsymbol{\Phi}=\left(\Phi_{1}, \ldots, \Phi_{N}\right)$ parameterizing that torus satisfy the motion equations of a free particle moving on a circle. These coordinates form, with their conjugate momenta $\mathbf{I}=\left(I_{1}, \ldots, I_{N}\right)$, a full set of phase space variables 
called "action-angle" variables. One of the results of the theorem is that the momenta $\mathbf{I}$ depend on constants of motion only $\mathbf{I}=\mathbf{I}(\mathbf{F})$. So, there exists a canonical transformation to the new variables $(\mathbf{p}, \mathbf{q}) \mapsto(\mathbf{I}, \boldsymbol{\Phi})$, in which the Hamiltonian depends on the constants of motion I (which are called action variables) only. Consequently, the equations of motion read

$$
\frac{d \mathbf{I}}{d t}=0, \quad \frac{d \mathbf{\Phi}}{d t}=\frac{\partial H(I)}{\partial \mathbf{I}} \quad\left\{I_{i}, \Phi_{j}\right\}=\delta_{i j}, \quad \Phi_{i} \in[0,2 \pi), \quad i, j=1, \ldots, N .
$$

Besides the practical importance, the action-angle formulation has an academic interest as well. From the academic viewpoint, it gives a precise indication of the (non)equivalence of different Hamiltonian systems. Indeed, gauging the integrable system by action-angle variables, we preserve the freedom only in the functional dependence of the Hamiltonian from the action variables, $H=H(\mathbf{I})$, and in the range of validity of the action variables, $I_{i} \in\left[\beta_{i}^{-}, \beta_{i}^{+}\right]$. Hence formulating the systems in terms of action-angle variables, we can indicate the (non)equivalence of different integrable systems. Let us refer, in this respect, to the recent paper 15, where, particularly, the global equivalence of $A_{2}$ and $G_{2}$ rational Calogero models, and their global equivalence with a free particle on the circle, has been established in this way.

In action-angle variables the Bohr-Sommerfeld quantization is equivalent to the canonical quantization, with a quite simple expression for the wavefunction

$$
\widehat{I}_{i} \Psi(\Phi)=I_{i} \Psi(\Phi), \quad \widehat{I}_{i}=-\imath \hbar \frac{\partial}{\partial \Phi_{i}}, \quad \Psi=\frac{1}{(2 \pi)^{N / 2}} \prod_{i=1}^{N} \mathrm{e}^{-\imath n_{i} \Phi_{i}}, \quad I_{i}=\hbar n_{i},
$$

where $n_{i}$ are integer numbers taking their values at the range $\left[\beta_{i}^{-}, \beta_{i}^{+}\right]$.

The general prescription for the construction of action-angle variables looks as follows [1. In order to construct the action-angle variables, we should fix the level surface of the Hamiltonian $\mathbf{F}=\mathbf{c}$ and then introduce the generating function for the canonical transformation $(\mathbf{p}, \mathbf{q}) \mapsto(\mathbf{I}, \boldsymbol{\Phi})$, which is defined by the expression

$$
S(\mathbf{c}, \mathbf{q})=\int_{\mathbf{F}=\mathbf{c}} \mathbf{p} d \mathbf{q},
$$

where $\mathbf{p}$ are expressed via $\mathbf{c}, \mathbf{q}$ by the use of the constants of motion. The action variables $\mathbf{I}$ can be obtained from the expression

$$
I_{i}(c)=\frac{1}{2 \pi} \oint_{\gamma_{i}} \mathbf{p} d \mathbf{q},
$$

where $\gamma_{i}$ is some loop of the level surface $\mathbf{F}=\mathbf{c}$. Then inverting these relations, we can get the expressions of $\mathbf{c}$ via action variables: $\mathbf{c}=\mathbf{c}(\mathbf{I})$. The angle variables $\mathbf{\Phi}$ can be found from the expression

$$
\mathbf{\Phi}=\frac{\partial S(\mathbf{c}(\mathbf{I}), \mathbf{q})}{\partial \mathbf{I}}
$$

In the next sections we will use the above formulae for the construction of the "action-angle" variables of the two-dimensional singular oscillator models.

\section{Singular Euclidean oscillator}

Let us demonstrate our approach with the simplest example of the singular oscillator on the two-dimensional Euclidean space, which is defined by the Hamiltonian (1.1). In polar coordinates this Hamiltonian reads

$$
H=\frac{p_{r}^{2}}{2}+\frac{p_{\varphi}^{2}+\alpha^{2}}{2 r^{2}}+\frac{\omega^{2} r^{2}}{2}, \quad x=r \cos \varphi, \quad y=r \sin \varphi .
$$

Taking into account that the angular momentum $p_{\varphi}$ is the constant of motion of this system, we can represent its generating function as follows: $S\left(p_{\varphi}, h, \varphi, r\right)=p_{\varphi} \varphi+\int_{H=h} p_{r} d r$. So, for the action variables we get the 
expressions

$$
I_{1}=\frac{1}{2 \pi} \oint p_{\varphi} d \varphi=p_{\varphi}, \quad I_{2}=\frac{1}{2 \pi} \oint p_{r} d r=\frac{h}{2 \omega}-\frac{\tilde{p}_{\varphi}}{2} \quad \text { where } \quad \tilde{p}_{\varphi} \equiv \sqrt{p_{\varphi}^{2}+\alpha^{2}}
$$

Respectively, the Hamiltonian takes the form

$$
H_{2 d}=\omega\left(2 I_{2}+\sqrt{I_{1}^{2}+\alpha^{2}}\right)
$$

The angle variables read

$$
\Phi_{1}=\varphi-\frac{p_{\varphi}}{2 \tilde{p}_{\varphi}} \arcsin \frac{\left(\widetilde{p}_{\varphi}+\omega r^{2}\right) \sqrt{2 h r^{2}-\widetilde{p}_{\varphi}^{2}-\omega^{2} r^{4}}}{\left(h+\widetilde{p}_{\varphi} \omega\right) r^{2}}, \quad \Phi_{2}=-\arcsin \frac{h-r^{2} \omega^{2}}{\sqrt{h^{2}-\widetilde{p}_{\varphi}^{2} \omega^{2}}} .
$$

For the reduction of this system to a one-dimensional one, we should put $p_{\varphi}=0$. In that case the Hamiltonian takes the form (where we replaced $r$ by $x) H_{1 d}=\omega\left(2 I_{2}+\alpha\right) \equiv 2 \omega \tilde{I}, \quad \tilde{I} \in[\alpha / 2, \infty)$. So, in the action-angle variable the one-dimensional singular oscillator is locally equivalent to the nonsingular one. The only difference is in the range of validity of the action variable. Hence, in spite of the close similarity in the action-angle variable formulation of the one- and two-dimensional singular oscillators, the dependence on the singularity term in the second system cannot be removed by the change in the range of validity of the action variable $\tilde{I}$, in contrast with the one-dimensional case. Let us notice that the action variable corresponding to the cyclic coordinate $\varphi$ coincides with the angular momentum $I_{1}=p_{\varphi}$. However, the respective angle variable $\Phi_{1}$ is different from the initial angle $\phi$. In other words, the "radial" motion, encoded in the dynamic of $I_{2}$ and $\Phi_{2}$ variables, has an essential impact on the "angular" motion. While the impact of $\varphi, p_{\varphi}$ variables in the radial motion is the shift $\alpha^{2} \rightarrow \alpha^{2}+p_{\varphi}^{2}$.

The inclusion of the constant magnetic field in the two-dimensional oscillator system does not essentially change its properties. Indeed, it is defined, in the two-dimensional planar system, by the potential

$$
\mathcal{A}=\frac{B_{0}}{2}(x d y-y d x)=\frac{B_{0} r^{2}}{2} d \varphi
$$

Hence, including the constant magnetic field in the two-dimensional singular oscillator, we shall get

$$
H=\frac{p_{r}^{2}}{2}+\frac{\left(p_{\varphi}-\frac{B_{0} r^{2}}{2}\right)^{2}}{2 r^{2}}+\frac{\alpha^{2}}{2 r^{2}}+\frac{\omega^{2} r^{2}}{2} \quad \Leftrightarrow \quad \widetilde{H}=\frac{p_{r}^{2}}{2}+\frac{\widetilde{p}_{\varphi}^{2}}{2 r^{2}}+\frac{\widetilde{\omega}^{2} r^{2}}{2},
$$

where we use the notation

$$
\widetilde{p}_{\varphi}^{2}=p_{\varphi}^{2}+\alpha^{2}, \quad \widetilde{\omega}^{2}=\omega^{2}+\frac{B_{0}^{2}}{4}, \quad \widetilde{H}=H+\frac{B_{0} p_{\varphi}}{2}
$$

Hence, we get the Hamiltonian (3.6). Thus, the impact of the magnetic field in the generating function $S\left(h, p_{\varphi}, r, \varphi\right)$ consists in the replacement (3.7). Respectively, the action variables and Hamiltonian are defined by the expressions

$$
I_{1}=p_{\varphi}, \quad I_{2}=\frac{\widetilde{h}}{2 \widetilde{\omega}}-\frac{\widetilde{p}_{\varphi}}{2} \quad \Rightarrow \quad H=\sqrt{\omega^{2}+\left(B_{0} / 2\right)^{2}}\left(2 I_{2}+\sqrt{I_{1}^{2}+\alpha^{2}}\right)-\frac{B_{0} I_{1}}{2} .
$$

The explicit expressions for angle variables reads

$$
\Phi_{1}=\varphi-\frac{p_{\varphi}}{2 \widetilde{p}_{\varphi}} \arcsin \frac{\left(\widetilde{p}_{\varphi}+\widetilde{\omega} r^{2}\right) \sqrt{2 \tilde{h} r^{2}-\widetilde{p}_{\varphi}^{2}-\widetilde{\omega}^{2} r^{4}}}{\left(\tilde{h}+\widetilde{p}_{\varphi} \widetilde{\omega}\right) r^{2}}, \quad \Phi_{2}=-\arcsin \frac{\widetilde{h}-r^{2} \widetilde{\omega}^{2}}{\sqrt{\widetilde{h}^{2}-\widetilde{p}_{\varphi}^{2} \widetilde{\omega}^{2}}} .
$$

It is seen that the magnetic field yields in the Hamiltonian the term linear on $I_{1}$, in addition to the predictable change of the effective frequency $\omega \rightarrow \sqrt{\omega^{2}+B_{0}^{2} / 4}$.

So, we constructed the action-angle variables for the two-dimensional singular oscillator in the constant magnetic field. In the next sections we shall consider a similar formulation for the models of singular spherical oscillators. 


\section{Singular Higgs oscillator}

In this Section we present the action-angle formulation of the singular Higgs oscillator (1.2). In our consideration we assume the unit radius of the sphere, $r_{0}=1$. The restoration of the the arbitrary radius can be carried out by the obvious redefinition of the Hamiltonian and the constants $\alpha, \omega$.

Since the angular momentum $p_{\varphi}$ is a constant of motion of the system, the the generating function of the action-angle variables takes the form

$$
S=p_{\varphi} \varphi+\int p_{\theta}\left(h, p_{\varphi}, \theta\right) d \theta
$$

where $H_{\text {Higgs }}=h$. From this generating function we get the action variables

$$
I_{1}=\frac{1}{2 \pi} \oint p_{\varphi} d \varphi=p_{\varphi}, \quad I_{2}=\frac{1}{2 \pi} \oint p_{\theta} d \theta=\frac{1}{\pi} \int_{\theta_{-}}^{\theta_{+}} \sqrt{2\left(h-\frac{p_{\varphi}^{2}}{2 \sin ^{2} \theta}-\frac{\alpha^{2}}{2} \cot ^{2} \theta-\frac{\omega^{2}}{2} \tan ^{2} \theta\right)} d \theta,
$$

where the integration limits $\theta_{ \pm}$are defined by the condition

$$
2 h=\frac{p_{\varphi}^{2}}{\sin ^{2} \theta_{ \pm}}+\alpha^{2} \cot ^{2} \theta_{ \pm}+\omega^{2} \tan ^{2} \theta_{ \pm}
$$

To calculate the integral in the second expression, we introduce the notation

$$
\xi=\frac{1}{a}[\cos 2 \theta+b], \quad a=\sqrt{1-2 \frac{p_{\varphi}^{2}+\alpha^{2}+\omega^{2}}{2 h+\alpha^{2}+\omega^{2}}+\left(\frac{p_{\varphi}^{2}+\alpha^{2}-\omega^{2}}{2 h+\alpha^{2}+\omega^{2}}\right)^{2}}, \quad b=-\frac{p_{\varphi}^{2}+\alpha^{2}-\omega^{2}}{2 h+\alpha^{2}+\omega^{2}} .
$$

In this terms the second integral in (4.11) reads (its value can be found by the use of standard methods, see, e.g. [16, 15])

$$
I_{2}=\frac{a^{2} \sqrt{2 h+\alpha^{2}+\omega^{2}}}{2 \pi} \int_{-1}^{1} \frac{\sqrt{1-\xi^{2}}}{1-(a \xi+b)^{2}} d \xi=\frac{1}{2}\left(\sqrt{2 h+\alpha^{2}+\omega^{2}}-\sqrt{p_{\varphi}^{2}+\alpha^{2}}-\omega\right) .
$$

Hence, the functional dependence of the Hamiltonian from the action variables is given by the expression

$$
H=\frac{1}{2}\left(2 I_{2}+\sqrt{I_{1}^{2}+\alpha^{2}}+\omega\right)^{2}-\frac{\alpha^{2}+\omega^{2}}{2} .
$$

For $\Phi_{1}$ and $\Phi_{2}$ we get

$$
\begin{gathered}
\Phi_{1}=\varphi-\frac{p_{\varphi}}{\widetilde{p}_{\varphi}} \arcsin \xi+\frac{p_{\varphi}}{\widetilde{p}_{\varphi}} \arctan \frac{1}{2 \widetilde{p}_{\varphi}}\left[\sqrt{\frac{\left(2 h-p_{\varphi}^{2}\right)^{2}-4 \omega^{2} \widetilde{p}_{\varphi}^{2}}{2 h+\alpha^{2}+\omega^{2}}}-\frac{2 h+2 \alpha^{2}+p_{\varphi}^{2}}{\sqrt{2 h+\alpha^{2}+\omega^{2}}} \frac{1+\sqrt{1-\xi^{2}}}{2 \xi}\right] \\
\Phi_{2}=-2 \arcsin \xi
\end{gathered}
$$

Here, as in the previous Section, we use the notation

$$
\widetilde{p}_{\varphi}=\sqrt{p_{\varphi}^{2}+\alpha^{2}}
$$

We presented the action-angle formulation of the singular Higgs oscillator (1.2) on the sphere of unit radius $r_{0}=1$. The action-angle formulation of the system on the sphere with arbitrary value of $r_{0}$ could be easily found from (4.14)-(4.17) by the replacement

$$
H_{r_{0}}=\frac{H}{r_{0}^{2}}, \quad \text { with } \quad \omega \rightarrow \omega r_{0}^{2} .
$$


In that case the Hamiltonian (1.2) is defined, in the action-angle variables, by the following expression:

$$
H=\frac{1}{2 r_{0}^{2}}\left(2 I_{2}+\sqrt{I_{1}^{2}+\alpha^{2}}+\omega r_{0}^{2}\right)^{2}-\frac{\alpha^{2}}{2 r_{0}^{2}}-\frac{\omega^{2} r_{0}^{2}}{2} .
$$

It is seen that, in the planar limit $r_{0} \rightarrow \infty$, it results in the Hamiltonian of the Euclidean singular oscillator (3.8) with $B_{0}=0$ (i.e. in the absence of constant magnetic field). However, the singular Higgs oscillator does not respect the inclusion of constant magnetic field, in contrast with the Euclidean one.

Indeed, the magnetic field which has a constant magnitude on the sphere, is the field of a Dirac monopole located at the center of sphere. It is defined by the following one-form:

$$
A_{D}=s(1-\cos \theta) d \varphi, \quad s=B_{0} r_{0}^{2} .
$$

Hence, the Hamiltonian of the singular Higgs oscillator interacting with a constant magnetic field, is defined by the expression

$$
H=\frac{p_{\theta}^{2}}{2 r_{0}^{2}}+\frac{\left[p_{\varphi}-s(1-\cos \theta)\right]^{2}}{2 r_{0}^{2} \sin ^{2} \theta}+\frac{\alpha^{2}}{2 r_{0}^{2}} \cot ^{2} \theta+\frac{\omega^{2} r_{0}^{2}}{2} \tan ^{2} \theta .
$$

Writing down the corresponding generating function we shall see that the impact of the magnetic field cannot be absorbed by the proper redefinition of constants. Hence, the inclusion of the magnetic field breaks the exact solvability of the (singular) Higgs oscillator, so that the presented model is not suitable for the study of the properties of spherical bands and length in the external magnetic field. However, this models is relevant for the consideration of their properties in the external potential, e.g. the electric field. Moreover, one can further modify the Higgs oscillator potential providing it by the anisotropy properties preserving the integrability of the system [12. Such a system would be useful to consider the quantum dots model restricted from the sphere to the spherical segment.

\section{$5 \quad$ Singular $C P^{1}$ oscillator}

There is another model of the spherical oscillator, which was introduced in [6] as a proper generalization of the oscillator system to the complex projective spaces. It was further generalized for the quaternionic projective spaces, as well [14. Its specific property was the respect of the constant magnetic fields. Since the complex projective plane is equivalent to the two- dimensional sphere, we can use this model for the definition of the two-dimensional magnetic oscillator. In spherical coordinates the potential of this alternative model of the spherical oscillator reads

$$
V_{C P^{1}}=2 \omega^{2} r_{0}^{2} \tan ^{2} \frac{\theta}{2} .
$$

In contrast with yje Higgs oscillator, it preserves the exact solvability property upon inclusion of the constant magnetic field. Respectively, its singular version, defined by the Hamiltonian (1.3) also remains exactly solvable in the presence of a constant magnetic field, at least, classically [13. Quantum mechanical solutions of the $C P^{1}$ singular oscillator are not constructed yet. But they could be found by a proper modification of the solutions of the corresponding non-singular system [6].

Inclusion of the constant magnetic field yields the following modification of the Hamiltonian (1.3):

$$
H=\frac{p_{\theta}^{2}}{2 r_{0}^{2}}+\frac{\left[p_{\varphi}-s(1-\cos \theta)\right]^{2}}{2 r_{0}^{2} \sin ^{2} \theta}+2 \omega^{2} r_{0}^{2} \tan ^{2} \frac{\theta}{2}+\frac{\alpha^{2}}{8 r_{0}^{2}} \cot ^{2} \frac{\theta}{2}, \quad s=B_{0} r_{0}^{2} .
$$

As before, we put, without loss of generality, $r_{0}=1$. The way of the restoring of $r_{0}$ is obvious. Then, in a completely similar way as in the previous cases, we can construct the action-angle variables of this system. For the action variables $I_{1}$ and $I_{2}$ we get

$$
I_{1}=p_{\varphi}, \quad I_{2}=\frac{1}{\pi} \int_{\theta_{-}}^{\theta^{+}} d \theta \sqrt{2 h-\frac{\left[p_{\varphi}-s(1-\cos \theta)\right]^{2}}{\sin ^{2} \theta}-4 \omega^{2} \tan ^{2} \frac{\theta}{2}-\frac{\alpha^{2}}{4} \cot ^{2} \frac{\theta}{2}}
$$


where $\theta_{ \pm}$are defined by the equation

$$
h=\frac{\left[p_{\varphi}+s\left(1-\cos \theta_{ \pm}\right)\right]^{2}}{2 \sin ^{2} \theta_{ \pm}}+2 \omega^{2} \tan ^{2} \frac{\theta_{ \pm}}{2}+\frac{\alpha^{2}}{8} \cot ^{2} \frac{\theta_{ \pm}}{2} .
$$

The explicit expression for the second integral looks as follows:

$$
\begin{gathered}
I_{2}=\frac{a^{2} \sqrt{2 h+4 \omega^{2}+\frac{\alpha^{2}}{4}+s^{2}}}{\pi} \int_{-1}^{1} \frac{\sqrt{1-\xi^{2}}}{1-(a \xi+b)^{2}} d \xi= \\
=\sqrt{2 h+s^{2}+\frac{\alpha^{2}}{4}+4 \omega^{2}}-\sqrt{\frac{p_{\varphi}^{2}+\alpha^{2}}{4}}-\sqrt{\left(\frac{p_{\varphi}}{2}-s\right)^{2}+4 \omega^{2}},
\end{gathered}
$$

where we introduced the notation

$$
\begin{gathered}
\xi=\frac{1}{a}[\cos \theta-b], \quad b=\frac{8 \omega^{2}+2 s^{2}-2 p_{\varphi} s-\frac{\alpha^{2}}{2}}{4 h+8 \omega^{2}+\frac{\alpha^{2}}{2}+2 s^{2}}, \\
a=\frac{2}{\sqrt{4 h+8 \omega^{2}+\frac{\alpha^{2}}{2}+2 s^{2}}} \sqrt{\frac{4 h^{2}-\left(8 \omega^{2}-p_{\varphi} s+2 s^{2}\right)\left(\frac{\alpha^{2}}{2}+p_{\varphi} s\right)}{4 h+8 \omega^{2}+\frac{\alpha^{2}}{2}+2 s^{2}}-\frac{p_{\varphi}^{2}}{2}+p_{\varphi} s .}
\end{gathered}
$$

Hence, from (5.5) we get that the explicit expression of the Hamiltonian has the following dependence from the action variables:

$$
H=\frac{1}{8}\left(2 I_{2}+\sqrt{I_{1}^{2}+\alpha^{2}}+\sqrt{\left(I_{1}-2 s\right)^{2}+16 \omega^{2}}\right)^{2}-\frac{s^{2}}{2}-\frac{\alpha^{2}}{8}-2 \omega^{2}
$$

The expressions for the angle variables look as follows:

$$
\begin{gathered}
\Phi_{1}=\varphi-\frac{1}{2}\left(\frac{p_{\varphi}}{\widetilde{p}_{\varphi}}+\frac{p_{\varphi}-2 s}{\sqrt{\left(p_{\varphi}-2 s\right)^{2}+16 \omega^{2}}}\right) \arcsin \xi+\frac{p_{\varphi}+s}{\sqrt{\left(p_{\varphi}-2 s\right)^{2}+16 \omega^{2}}} \arctan \eta_{+}-\frac{p_{\varphi}}{\widetilde{p}_{\varphi}} \arctan \eta_{-}, \\
\Phi_{2}=\frac{\partial S}{\partial I_{2}}=-\arcsin \xi .
\end{gathered}
$$

Here we used the notation

$$
\widetilde{p}_{\varphi} \equiv \sqrt{p_{\varphi}^{2}+\alpha^{2}}, \quad \eta_{ \pm} \equiv \frac{(1 \pm b)\left(\frac{1}{\xi}+\sqrt{\frac{1}{\xi^{2}}-1}\right) \pm a}{\sqrt{(1 \pm b)^{2}-a^{2}}}
$$

Finally, let us restore the radius $r_{0}$ performing the replacement (4.19). In that case the Hamiltonian (5.2) is expressed via action variables as follows:

$$
H=\frac{1}{8 r_{0}^{2}}\left(2 I_{2}+\sqrt{I_{1}^{2}+\alpha^{2}}+\sqrt{\left(I_{1}-2 B_{0} r^{2}\right)^{2}+16 \omega^{2} r_{0}^{4}}\right)^{2}-\frac{B_{0}^{2} r_{0}^{2}}{2}-\frac{\alpha^{2}}{8 r_{0}^{2}}-2 \omega^{2} r_{0}^{2}
$$

It is seen that, in the planar limit $r_{0} \rightarrow \infty$, it results in the Hamiltonian of the Euclidean singular oscillator (3.8).

So, we presented the action-angle formulation of the model of the spherical singular oscillator interacting with a constant magnetic field (5.2). The Hamiltonian of the model is non-degenerate on both action variables. But it depends on these variables via elementary functions in the presence of a constant magnetic field.

These tell us the area of application of the Higgs oscillator potential and of the $C P^{1}$ oscillator one. The Higgs model is useful for the behavior of the quantum dots systems in the external potential field, e.g, in the electric field. The $C P^{1}$ model should be applied for the study of the behavior of a spherical quantum dots model in the external magnetic field. 


\section{Conclusion}

In this paper we presented the "action-angle" formulation of the textbook two-dimensional singular oscillator model, and of its two spherical generalizations, based on the so-called Higgs and $C P^{1}$ spherical oscillator potentials. Writing this paper we had in mind two goals: the first was the suggestion to use the action-angle variables in the study of quantum dots models. Another goal was the suggestion of singular spherical oscillator models to the role of confinement potentials in spherical quantum rings. In the study of spherical rings we suggested, for the role of the constant magnetic field, the magnetic field of the Dirac monopole located at the center of the sphere. Surely, the Dirac monopole is a non-physical object. However, since we assume to use it for the description of the particles localized on a part of the sphere, the non-physical nature of the Dirac monopole can be ignored. The monopole can be considered, e.g. as a pole of the magnetic dipole. The possible impact of the Dirac monopole on the properties of quantum dots model has been considered, e.g., in [17. Besides, magnetic monopoles emerge as a class of magnets known as spin ice [18.

We restricted ourselves to the formal mathematical formulation of the systems, postponing the consideration of the particles behavior in the spherical quantum rings, described by the use of the above presented models, for the future study.

Acknowledgments. We are grateful to Tigran Hakobyan and Haik Sarkisyan for useful discussions and comments. The work was supported by and ANSEF-2229PS grant and by Volkswagen Foundation grant $\mathrm{I} / 84496$.

\section{References}

[1] V. I. Arnold, Mathematical methods in classical mechanics, Nauka Publ., Moscow, 1973

[2] H. Goldstein, Classical Mechanics, Addison-Wesley Press, Cambridge, 1950

[3] V. Ya. Prinz et al, Phisica E6, 828 (2000); Nanotechnology 12, 399 (2001);

O. G. Schmidt, K. Eberl, Nature 410,168(2001)

[4] D. Leonard et al. Appl. Phys. Lett.63, 3203 (1993); Phys. Rev.B50, 11687 (1994)

[5] P. W. Higgs, J. Phys.A12, 309 (1979) ;

H. I. Leemon, J. Phys. A12 (1979) 489.

[6] S. Bellucci and A. Nersessian, Phys. Rev. D 67, 065013 (2003) [Erratum-ibid. D 71, 089901 (2005)]

S. Bellucci, A. Nersessian, A. Yeranyan, Phys. Rev. D70, 045006 (2004); Phys. Rev. D70, 085013 (2004); S. Bellucci, A. Nersessian, A. Yeranyan, Phys. Rev. D74, 065022 (2006); S. Bellucci, A. Nersessian, (2003) Supersymmetric Kahler oscillator in a constant magnetic field Contributed to International Seminar on Supersymmetries and Quantum Symmetries SQS 03, Dubna, Russia, 24-29 Jul 2003 arXiv:hep-th/0401232.

[7] A. Lorke et al, Phys. Rev. Lett. 84, 2223 (2000)

A. Fuhrer et al, Nature 413, 822 (2001)

[8] T. Chakraborty and P. Pietlainen, Phys. Rev.B50, 8460 (1994)

[9] W.-C. Tan and J.C.Inkson, Semicon.Sci.Technology, 11, 1635 (1996)

[10] J. Simonin, C. R. Proetto, Z. Barticevic, G. Fuster, Phys. Rev. B70, 205305 (2004) ;

[11] A. K. Atayan et al,J. Comput. Theor. Nanosci. 7, 1165(2010);

[12] A. Nersessian, V. Yeghikyan, J. Phys. A41 155203 (2008) . 
[13] K. S. Aramyan, Theor. Math. Phys.156, 1075(2008)

[14] L. Mardoyan and A. Nersessian Phys. Rev. B72, 233303 (2005);

S. Bellucci, L. Mardoyan, A. Nersessian Phys. Lett. B636, 137 (2006)

[15] O. Lechtenfeld, A. Nersessian, V. Yeghikyan, "Action-angle variables for dihedral systems on the circle" arXiv:1005.0464] [hep-th]

[16] G. M. Fichtenholz, Foundations of Differential and Integral Calculus, 6th edition, 1966, Nauka Publ, Moscow,(in Russian)

[17] L. G. Mardoyan, L. S. Petrosyan and H. A. Sarkisyan, Phys. Rev. A68, 014103 (2003)

L. Mardoyan et al.J. Phys. A 40, 5973 (2007) S. Bellucci, V. Ohanyan Phys. Lett. A372, 5765 (2008).

[18] C. Castelnovo, R. Moessner, S. L. Sondhi, Nature, 451, 42 (2008). 